\title{
Diabetes mellitus tipo 1 no contexto familiar e social
}

\author{
Diabetes mellitus type 1 in the familiar and social context
}

Anna Paula P. Góes ${ }^{1}$, Maria Rita R. Vieira², Raphael Del Roio Liberatore Júnior ${ }^{3}$

\section{RESUMO}

Objetivo: Identificar as dificuldades da criança com diabetes mellitus tipo 1 no convívio diário com familiares e com a sociedade, enfocando aspectos relativos à alimentação e ao tratamento.

Métodos: Estudo transversal, realizado por meio de questionário semi-estruturado aplicado a pais e crianças com diabetes mellitus tipo 1, acompanhados pelo Grupo de Diabetes mellitus do Ambulatório de Pediatria da Faculdade de Medicina de São José do Rio Preto (Famerp), São Paulo. Foram estudadas 13 crianças, coletando-se dados demográficos e aspectos relativos às dificuldades no controle da doença.

Resultados: As características do grupo de crianças estudadas foram: gênero feminino (77\%), idade entre oito e 12 anos (77\%), brancas (77\%), católicas (61\%) e com antecedentes familiares de diabetes (54\%). Como dificuldades foram citadas: o custo de alimentos especiais; o medo do desconhecido; ter que aprender sobre a doença rapidamente; ter vergonha de ter diabetes. Do total de crianças, $23 \%$ estavam aprendendo a aplicar insulina e $46 \%$ dos pacientes seguiam a orientação alimentar. A mãe foi o parente mais próximo que auxiliava a criança no tratamento em $69 \%$ dos casos. A hiperglicemia foi relatada por $62 \%$ das crianças, sendo detectada apenas após a realização da glicemia capilar.

Conclusões: $\mathrm{O}$ presente trabalho salienta a importância de campanhas para orientar os pais sobre o controle da doença, os fatores associados ao desenvolvimento da doença e a necessidade de identificar os sintomas de hiperglicemia e a terapêutica alimentar, a fim de minimizar riscos futuros.

Palavras-chave: criança; adolescente; diabetes mellitus; assistência domiciliar.

\section{ABSTRACT}

Objective: To identify the difficulties of children with diabetes mellitus type 1 in their family and social daily life, focusing on aspects related to feeding and treatment.

Methods: This cross-sectional study was performed with a semi-structured questionnaire applied to parents and children with diabetes mellitus type 1 at the Diabetes Clinic of the São José do Rio Preto Medical, São Paulo, Brazil. The interview of 13 children included questions regarding demographic data and difficulties related to the control of the disease.

Results: The following characteristics were found among the studied children: female gender in $77 \%$, age between eight and 12 years in $77 \%$, white race in $77 \%$, catholics in $61 \%$ and presence of family history of diabetes in 54\%. Regarding the disease control, the following difficulties were mentioned: special food cost, fear of the unknown, need for quick learning about the disease and shame of having diabetes. Out of 13 children, 23\% were learning how to apply insulin and $46 \%$ followed the diet prescription at the time of the interview. In $69 \%$ of cases, the mother was the closest relative that has helped the child to manage the treatment. Hyperglycemia was present in $62 \%$ of the children, and it was detected only after capillary glycemia test.

Conclusions: The present study points out the importance of campaigns to guide parents about the disease control, about factors associated with the development of the disease and the need for identifying the hyperglycemia symptoms in order to minimize future complications of the diabetes.

Key-words: child; adolescent; diabetes mellitus; home nursing.

\footnotetext{
'Acadêmica de Enfermagem da Faculdade de Medicina de São José do Rio Preto (Famerp)

2Mestre e doutoranda em Ciências da Saúde pela Famerp

${ }^{3}$ Doutor em Pediatria pela Universidade de São Paulo (USP)

Endereço para correspondência:

Anna Paula P. Góes
}

Rua São Paulo, 510 - Jardim Bordon

CEP 15060-035 - São José do Rio Preto/SP

E-mail: annappg@yahoo.com.br

Recebido em: 2/1/2007

Aprovado em: 5/4/2007 


\section{Introdução}

$\mathrm{O}$ diabetes mellitus (DM) é uma doença do metabolismo, caracterizada por deficiência total ou parcial do hormônio insulina $^{(1,2)}$. É resultante da adaptação metabólica ou alteração fisiológica em quase todas as áreas do organismo. Trata-se do distúrbio mais freqüente da infância, verificando-se uma incidência máxima no início da adolescência ${ }^{(1,3)}$.

ODM está associado ao aumento da mortalidade e ao alto risco de desenvolvimento de complicações micro e macrovasculares, bem como neuropatias. É causa de cegueira, insuficiência renal e amputações de membros, sendo responsável por gastos expressivos em saúde, além de substancial redução da capacidade de trabalho e da expectativa de vida ${ }^{(4)}$.

Os objetivos do tratamento devem estar claros a todos os membros da equipe e para os familiares. Assim, de imediato, interessa o alívio dos sintomas da descompensação diabética por meio da ação de um sistema de saúde apto a reconhecer, diagnosticar e iniciar o tratamento de emergência. Em médio prazo, interessa a aquisição da normoglicemia, com vida social aceitável por meio de educação adquirida com equipe multiprofissional especializada. Em longo prazo, objetiva-se evitar ou reduzir as complicações crônicas (retinopatia, neuropatia, nefropatia, aterosclerose), mantendo-se a normoglicemia, a qual depende principalmente do grau do autocuidado do próprio paciente ${ }^{(5)}$.

O tratamento do DM interfere no estilo de vida, é complicado, doloroso, depende de autodisciplina e é essencial à sobrevida. A abordagem terapêutica envolve vários níveis de atuação, como a insulinoterapia, a orientação alimentar, a aquisição de conhecimentos sobre a doença, a habilidade de auto-aplicação da insulina e o autocontrole da glicemia, a manutenção da atividade física regular e o apoio psicossocial ${ }^{(5)}$.

Sabe-se que a família e os amigos influenciam no controle da doença quanto ao seguimento do tratamento, da dieta e na participação em um programa regular de exercícios ${ }^{(6,7)}$. Pelo fato de interferir na intimidade do núcleo familiar, a dieta é freqüentemente motivo de dificuldades e falta de adesão(5). As crianças com diabetes não precisam de nenhum alimento ou suplemento especial, mas de calorias suficientes para equilibrar o consumo diário de energia e suprir as necessidades para seu crescimento e desenvolvimento ${ }^{(1)}$.

Com base nas alterações de estilo de vida impostas pelo controle do diabetes mellitus tipo 1, este trabalho teve por objetivo identificar as principais dificuldades na vivência familiar e social de crianças com a referida doença, enfocando os aspectos da alimentação, do tratamento e das relações em sociedade.

\section{Métodos}

Estudo transversal e descritivo, realizado entre outubro de 2005 e novembro de 2006 com crianças diabéticas acompanhadas no serviço de endocrinologia pediátrica. Das 107 crianças atendidas no referido serviço, 17 residiam em São José do Rio Preto, São Paulo, e foram convidadas para participar do trabalho. Destas, quatro pais não aceitaram participar do estudo; portanto, foram incluídas 13 crianças.

O protocolo de pesquisa foi aprovado pelo Comitê de Ética em Pesquisa da Faculdade de Medicina de São José do Rio Preto (Famerp) e os dados armazenados em planilha eletrônica (Excell 97).

A coleta de dados foi realizada por meio de entrevista semiestruturada com questões abertas e fechadas, no domicílio das crianças estudadas. O questionário apresentava perguntas sobre a identificação do paciente (idade, gênero, raça, escolaridade, religião, bairro onde reside), antecedente familiar de diabetes, tempo de diagnóstico, uso de insulina e quem aplicava a medicação, existência de orientação nutricional prévia, comportamento da família em relação à mudança na alimentação, principais mudanças sentidas pela família e pela criança em relação à doença e o conviver com a mesma após seu diagnóstico, além das principais mudanças e dificuldades nas famílias com mais de um filho. Foi perguntado também se a criança era capaz de reconhecer os sintomas de hipo e hiperglicemia, a freqüência de internações relacionadas ao diabetes e a causa destas e, finalmente, se a criança realizava atividade física e com qual freqüência.

\section{Resultados}

Foram incluídas no estudo 13 crianças com média de idade de

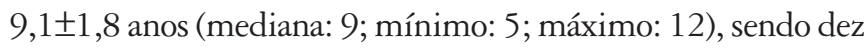
crianças do gênero feminino, dez de cor branca, oito seguiam a religião católica e todas freqüentavam escola. Das 13 crianças, 11 possuíam irmãos, 11 conviviam com famílias constituídas de até cinco pessoas e sete crianças possuíam antecedentes familiar de diabetes. O tempo de diagnóstico foi, em média, 3,2 22,5 anos (mediana: 3; variação: um mês a nove anos).

Quando questionado quanto à mudança de comportamento de pessoas próximas a criança com o diagnóstico de DM tipo 1, verificou-se que a família se mobilizou para contribuir positivamente no controle da glicemia (cinco famílias). Os irmãos mais velhos apresentaram ciúmes pelo fato de a doença exigir uma atenção maior para seu controle (uma criança). 
Em relação às crianças com diabetes, uma criança apresentou revolta por estar anteriormente saudável e depois ter desenvolvido uma doença crônica, com necessidade de cuidados especiais por toda a vida. Outra criança se recusava a voltar à escola por causa do diagnóstico e uma criança se recusou a assumir o fato de ser portadora de uma doença crônica. Houve também o relato de insegurança quanto a mudanças de hábito para tentar a estabilidade da glicemia e a adaptação à vida com a criança em tratamento contínuo.

Além disso, cinco responsáveis citaram que, durante o dia, as crianças permaneciam com algum parente, em geral a avó.

Com relação ao número de internações, verificou-se que cinco das 13 crianças apresentaram uma internação hospitalar por ocasião do diagnóstico da doença. Como motivos para tais internações foram alegados: perda da consciência precedida de sinais de moleza da criança; excesso de fome, sede e diurese; emagrecimento rápido; crise convulsiva por hipoglicemia.

Em relação à aplicação de insulina, quatro das 13 crianças aplicavam a medicação por si próprias, uma vez ao dia. Quando havia necessidade de novas doses de insulina em outros horários, a aplicação era realizada, em geral, pela mãe. Das 13 crianças estudadas, três estavam em fase de aprendizado quanto à aplicação da insulina, com supervisão de um responsável, e relatavam sentir medo da dor ao realizar o procedimento. A mãe foi citada por $69 \%$ das crianças como a pessoa que assumia todo o tratamento.

Em relação às possíveis dificuldades referidas pela criança e por responsáveis com o desenvolvimento do DM tipo 1, foram citados o fato de que alimentos voltados ao diabético são mais caros (uma família); medo do desconhecido (duas crianças); ter que aprender rapidamente sobre a doença, o seu tratamento e a dosagem de insulina, bem como a forma de sua aplicação (duas crianças); a família ter que lidar com o caso de uma criança ter desenvolvido o DM tipo 1 e, muitas vezes, a mãe ter que ficar "furando" o filho (seis famílias); a criança ter vergonha de contar que é diabética (uma criança); receio de que o filho sofra preconceito por ser diabético (uma família).

$\mathrm{Na}$ caracterização dos sintomas de hipoglicemia, três crianças referiam tremores, fome e dor de cabeça; quatro apresentaram fraqueza; uma sonolência; duas haviam perdido a consciência; uma referiu ter sentido tontura; uma mencionou dor no estômago e uma referiu choro. Tais crianças, quando sentiam que estavam com hipoglicemia, tomavam alguma bebida doce, como refrigerantes, leite com achocolatado ou com açúcar ou comiam pão.
Em relação à hiperglicemia, oito crianças referiram que tal diagnóstico só era feito após a realização da glicemia capilar, não sendo descrito nenhum sintoma específico quando a glicemia estava elevada. As demais crianças citaram como sintomas de hiperglicemia: dor de cabeça, sudorese em mãos, sede e aumento do volume de urina, agressividade, fome e dor no estômago. Quando as crianças estavam na condição de hiperglicemia, foi referido que as mães aplicavam insulina ou os responsáveis orientavam a criança a se acalmar, mandavam a criança brincar, levavam à Unidade Básica de Saúde ou davam orientações dietéticas diversas.

Em relação à orientação alimentar, dez das 13 crianças relataram tê-la recebido e, dessas, seis referiram seguir parcialmente tal orientação. A participação da família na mudança de hábito alimentar da criança foi citada por oito das 13 famílias. Quando questionados a respeito das atividades físicas, constatou-se que nove crianças participavam de Educação Física na escola (duas a três vezes por semana), quatro das 13 crianças participavam de escolinha de Futebol (duas a três vezes por semana), uma dançava balé (duas vezes por semana) e uma caminhava três a quatro vezes por semana.

\section{Discussão}

O diagnóstico de diabetes em uma criança é motivo de ansiedade no núcleo familiar por se tratar de uma doença crônica, de etiologia incerta e com possíveis complicações futuras ${ }^{(5)}$.

A vida da criança e da família passa a ser regida pela doença, com exames, internações e viagens, pois muitos residem em outras localidades. O primeiro impacto surge por ocasião do diagnóstico da doença ${ }^{(8)}$.

Sabe-se que as limitações experimentadas pela criança com diabetes são inúmeras e desencadeiam vários sentimentos, como o medo e a insegurança, além de atitudes que vão do conformismo ao autocuidado $^{(3)}$. No presente estudo, houve relato de crianças que se adaptaram à restrição alimentar e à realização da atividade física, rejeitando a aplicação de insulina com receio de dor.

É importante que a família compreenda a natureza de qualquer alteração de saúde e as implicações sobre os seus membros. Se um dos indivíduos necessita adquirir novos conhecimentos ou novas habilidades para manter a saúde, tais como medicamentos a serem tomados, é importante que um familiar participe, pois poderá ajudar a assumir os cuidados com o paciente quando esse adoecer ou ficar incapacitado ${ }^{(9)}$. 
Acredita-se que, a partir do momento em que a família começa a conviver com a criança doente e se envolver com os cuidados diários, os sentimentos de medo, negação e desespero acabam sendo transformados em aceitação ${ }^{(10)}$. Durante a coleta de dados, alguns responsáveis referiram que a não aceitação inicial foi pelo fato da criança ter desenvolvido o DM, mas que, atualmente, a mudança de hábito ocorreu na tentativa de controle da glicemia da criança e por não querer que esta tivesse vontade de alimentos que não pode ingerir.

Para conseguir um bom controle do diabetes, é importante a educação tanto do paciente quanto da família, deixando explícito que esta deve estar envolvida no tratamento tão logo tenha passado o primeiro impacto do diagnóstico ${ }^{(11)}$.

Em relação à terapia medicamentosa, é importante haver o estímulo à auto-aplicação de insulina e, para tal, não se deve levar em conta apenas a idade da criança. Deve-se analisar também o conhecimento da criança sobre a doença, o desenvolvimento cognitivo, o desempenho apresentado no controle de atividades relacionadas ao diabetes e o ambiente familiar para decidir como e quando transferir esta responsabilidade para a criança ${ }^{(11,12)}$. Diante disso, é válido inferir a importância de um maior incentivo à auto-aplicação de insulina.

É importante deixar claro para as crianças quais são os sintomas característicos de hipo e hiperglicemia. Como sintomas de hipoglicemia podem ser citados: sensação de fome aguda, dificuldade para raciocinar, sensação de fraqueza com um cansaço muito grande, sudorese exagerada, tremores finos ou grosseiros de extremidades, bocejamento, sonolência, visão dupla, confusão que pode caminhar para a perda total da consciência, ou seja, coma ${ }^{(13)}$. Com o estudo, percebeu-se que as crianças não apresentaram dificuldade significativa em relação à hipoglicemia.

Além disso, é válido ressaltar a importância de orientar a família para garantir o reconhecimento rápido e correto dos sintomas de hipo ou hiperglicemia a fim de se evitar complicações para a criança, redução no número de internações.

Os principais sintomas de hiperglicemia são: aumento da sede (polidipsia), excesso de urina (poliúria), muita fome (polifagia) e emagrecimento. Outros sintomas são: sonolência, dores generalizadas, formigamentos e dormências, cansaço doloroso nas pernas, câimbras, nervosismo, indisposição para o trabalho, desânimo, turvação da visão, cansaço físico e mental $^{(7)}$. Em relação à hiperglicemia, torna-se importante educar melhor as crianças e suas famílias quanto os principais sinais e sintomas da mesma, uma vez que o DM é uma doença crônica, que a médio e longo prazo pode trazer conseqüências como a retinopatia diabética.

Em crianças no primeiro ano de vida, a irritabilidade, o desconforto, a agitação e mesmo o torpor ou coma são interpretados erroneamente e o diagnóstico de DM pode não ficar evidente, em um primeiro momento ${ }^{(5)}$. Diferente de adultos, o diagnóstico na criança é, em geral, suspeitado diante de manifestações clínicas. A hiperglicemia, na maioria das vezes bastante acentuada, provoca glicosúria e diurese osmótica, com conseqüente desidratação ${ }^{(14)}$. A necessidade de compensar a desidratação leva à modificação dos hábitos desse paciente, trazendo de volta a enurese noturna e provocando poliúria e polidipsia. $\mathrm{O}$ intenso catabolismo e a perda eletrolítica levam a emagrecimento, fraqueza e desânimo, que podem ser acompanhados de hipoxemia em substituição à clássica polifagia, especialmente nos casos associados à infecção ${ }^{(14)}$.

Sabe-se que a família e os amigos influenciam no controle da doença quanto ao seguimento do tratamento, da dieta e na participação em um programa regular de exercícios. Um estudo demonstrou que pacientes com diabetes mellitus que tiveram apoio adequado de amigos e familiares aderiram melhor às orientações de autocuidado ${ }^{(7)}$. Tal fato demonstra a importância da aceitação da doença pela criança e sua família.

A doença acarreta mudanças significativas na relação que o paciente diabético estabelece com seu próprio corpo e com o mundo que o cerca. $\mathrm{O}$ conflito entre o desejo de se alimentar e a necessidade imperiosa de contê-lo está sempre presente na vida cotidiana do mesmo ${ }^{(15)}$. Os benefícios da prática da atividade física regular para a saúde têm sido amplamente documentados. Há várias evidências de que os resultados inicialmente obtidos em programa de atividade física só serão mantidos se os indivíduos continuarem praticando exercício apropriado em longo prazo ${ }^{(16)}$. No presente estudo, verificou-se que as famílias não apresentaram problemas em relação à prática de atividade física, uma vez que as mesmas procuraram colocar regularmente as crianças em atividades preferidas pelas próprias crianças.

A melhora nas medidas fisiológicas, tais como a redução de triglicérides e do colesterol LDL, o aumento do colesterol HDL, a diminuição da freqüência cardíaca em repouso e em atividade e a redução da pressão arterial, entre outras, decorrentes de um estilo de vida fisicamente ativo, são ainda mais importantes nos portadores de diabetes mellitus, uma vez que o risco de mortalidade por doenças coronarianas é quatro a cinco vezes maior nesses indivíduos ${ }^{(16)}$. 
Os resultados obtidos neste estudo mostram que, desde o momento em que é levantada a hipótese de DM tipo 1 até a sua confirmação diagnóstica e o início da convivência com a doença, houve insegurança na população estudada, muitas vezes devido ao desconhecimento sobre a doença e sua repercussão no cotidiano da família.

Apesar dessas dificuldades, grande parte das crianças não necessitou de internações hospitalares no período estudado, fato que ocorreu apenas por ocasião do diagnóstico da doença.

\section{Referências bibliográficas}

1. Wong DL. A criança com disfunção endócrina. In: Wong DL, editor. Enfermagem pediátrica: elementos essenciais à intervenção efetiva. $5^{\mathrm{a}}$ ed. Rio de Janeiro: Guanabara Koogan; 1999. p. 936-49.

2. Santos JR, Enuro SRF. Adolescentes com Diabetes mellitus tipo 1: seu cotidiano e enfrentamento da doença. Psicol Reflex Crit 2003;16:411-25.

3. Moreira PL, Dupas G. Vivendo com o diabetes: a experiência contada pela criança. Rev Latino Am Enfermagem 2006;14:25-32.

4. Assunção MC, Santos IS, Gigante DP. Atenção primária em diabetes no sul do Brasil: estrutura, processo e resultado. Rev Saude Publica 2001;35:88-95.

5. Setian N, Damiani D, Dichtchekenian V, Manna TD. Diabetes mellito. In: Marcondes E, Vaz FAC, Ramos JLA, Okay Y, editores. Pediatria básica. $9^{a}$ ed. São Paulo: Sarvier; 2003. p. 382-92.

6. Pace AE, Nunes PD, Ochoa-Vigo K. O conhecimento dos familiares acerca da problemática do portador de diabetes mellitus. Rev Latino Am Enfermagem 2003;11:312-9.

7. Marcelino DB, Carvalho MDB. Reflexões sobre o Diabetes Tipo 1 e sua relação com o emocional. Psicol Reflex Crit 2005;18:72-7.

8. Vieira MA, Lima RA. Crianças e adolescentes com doença crônica: convivendo com mudanças. Rev Latino Am Enfermagem 2002;10:552-60.
Isto indica que, apesar das dificuldades existentes, pode-se considerar que o acompanhamento e o plano de cuidados da criança foram satisfatórios.

É válido ressaltar que, em muitos casos, os sintomas de hiperglicemia ainda não estavam claros para os envolvidos com o tratamento, uma vez que esta situação era percebida apenas com a glicemia capilar. Esse fato mostra a importância de orientar os familiares e as crianças sobre as principais características da hiperglicemia para evitar complicações futuras para a criança.

9. Potter PA, Perry AG. O contexto familiar de enfermagem. In: Potter PA, Perry AG, editores. Grande tratado de enfermagem prática clínica e prática hospitalar. $3^{a}$ ed. São Paulo: Santos; 2002. p. 313-21.

10. Zanetti MA, Mendes IAC. Dificuldades apresentadas por mães de crianças e de adolescentes diabéticos tipo 1, antes do estabelecimento do diagnóstico. Acta Paulista Enfermagem 2002;15:17-23.

11. Antonia CD, Zanetti ML. Auto-aplicação de insulina em crianças portadoras de Diabetes Mellitus tipo 1. Rev Latino Am Enfermagem 2000;8:51-8.

12. Ataide MBC, Damasceno MMC. Fatores que interferem na adesão ao autocuidado em diabetes. Rev Enfermagem UERJ 206;14:518-23.

13. Sociedade Brasileira de Diabetes [homepage na internet]. Hipoglicemia. [citado em 15 de outubro de 2006]. Disponível em: http://www.diabetes.org.br/diabetes/hipog.php

14. Silva IN, Chagas AJ. A criança diabética. In: Amaral CFS, Pedroso ERP, Fonseca JGM, Rocha MOC, Couto RC, Leal SS et al, editores. Enciclopédia da saúde. Diabetes mellitus. $2^{a}$ ed. Rio de Janeiro: Medsi; 2002. p. 425-33.

15. Santos EC, Zanetti ML, Otero LM, Santos MA. O cuidado sob a ótica do paciente diabético e de seu principal cuidador. Rev Latino Am Enfermagem 2005;13:397-406.

16. Fechio JJ, Malerbi FE. Adesão a um programa de atividade física em adultos portadores de diabetes. Arq Bras Endocrinol Metab 2004;48:267-75. 